\title{
Lineamenti del progetto di un veicolo per una missione lunare completa $\left({ }^{*}\right)$
}

\author{
G. Costerse $(* *)$ \\ Riceruto il is Magnio lati3
}

Reassinto. - Nel presente lavoro si studiano, con lanusilio di una formulazione approssimata. le fasi puramente gravitazionali della missione: ricavate da questo studio le condizioni a limiti sulle relocita per le fasi pro. pulse, si considerano le equazioni di queste e si perviene, sulli scorta anche di precedenti lavori, alla relativa espressione dei rapporti di massa.

Acelarate delle limitazioni di principio nelle prestazioni dei monostadi, si estudiata lia ripartizione del vettore in istali, pervenento a delle utili formule per il progetto. Lapplicazione di queste formule fornisce dei risultati numerici che sono l'ougretto di mal prima discussione.

Sumarx. - In this work, on the hasis of an approximate formulation, the merely gravitational phases of the mission are investigated: after having stated in this way the boundary conditions for the propelled phases, the re lated equations are considered in order to reach proper expressions for milss ratio.

Missile division in stages is the further ain of the work: the stuty fur. nishes some useful design formulas. By applying these formulas some basic results are stated and discusised.

\section{1. - INTRODVZIONE.}

Per una missione lunare completa somo state recentemente proposte

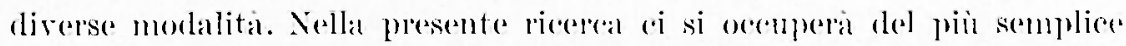
di questi modelli, rioc di quello che comsta di quattro fasi propulse a di due puramente erravitazionali.

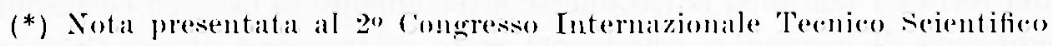
dello spazio, Roma. 19-23 (iingno 1962.

(**) Lstituto di Aeronautiea - Vniversita di Sapoli. 
Tralasciando gli elementi divulgativi, sottolineamo subito l'importanza di principio che hanno, per il progetto di un veicolo adleguato alla missione, le fasi puramente gravitazionali della traiettoria, poiche proprio lo studio di queste dovra fornire le condizioni ai limiti sulle velocita per le equazioni differenziali del moto nelle fasi propulse.

E il caso di rilevare anche che il campo gravitazionale dovuto alla terra ed al suo satellite naturale crea una correlazione, che l'analisi successiva precisera quantitativamente, fra tali condizioni ai limiti. Questa relazione, che va riguardata come una vera e propria legge naturale, impronta i fondamenti del progetto dei veicoli per la missione in esane.

Essa difatti mostrera che non e possibile giungere in prossimita della superficie lunare con valori arbitrariamente piccoli della velocita, come sarebbe desiderabile per ragioni di sicurezza e di economia di massa all'atterraggio. In altri termini si dimostrera che esiste un limite inferiore per la velocita di impatto non frenato.

In piu si dimostrera che questa velocita e prossima a quella di evasione dalla superficie lunare, realizzandosi una condizione molto restrittiva con riferimento alle tolleranze da applicarsi alle fasi propulse.

Prima di entrare nei dettagli della trattazione vogliamo precisare l'ambito entro oui il presente lavoro verra mantenuto. Si rerchera di delineare il progetto di un veicolo per la nissione in esame, indagando sui seguenti aspetti, a nostro avviso fondamentali e in larga misura indipendenti dagli altri che non verranno esaminati:

- deteminazione del rapporto di massa globale;

- questioni relative alla ripartizione del veicolo in stadi;

- criteri per assegnare le relative dimensioni di ingombro.

L'esame dei punti predetti, se e ben lungi dall'esaurire il progetto. del veicolo in esame, ne costituisce tuttavia un"ossatura che difficilmente altri fattori possono modificare. Difatti, aspetti che qui non verranno trattati, quali, ad esempio, la protezione dei missili da radiazioni, si traduranno in definitiva in aumenti del " carico utile » ed eventualments dei valori dei rapporti strutturali.

\section{2. - StUdio DELLE FASI PURAMENTE GRAMTTAZIONALI.}

Il problema del moto di un veicolo nel campo gravitazionale dovuto alla terra ed alla luna si riconnette strettamente al rlassico erl insolubile problema di meccanica celeste del moto di tre corpi attraentisi newtonianamente. 
Ena formulazione approssimata che a prima vista risulta conveniente e quella relativa al cosiddetto problema ristretto dei tre corpi. Ma in questa formulazione $v^{-9}$ e una limitazione che la rende inaccettabile ai nostri fini: i centri dei tre corpi devono muorersi in un piano.

Le inclinazioni del piano di rotazione della luna attorno alla terian a dell'asse di rotazione rerrestre sul piano dellecelittica creano una configurazione tale da renclere difficile, da un punto di vista dinamico, inserire il mobile nel piano dell'eclitticar.

Sotto altri aspetti, al fine di rintraceiare delle proplieta genemali della traiettoria, la formulazione del problema ristretto dei tre corpi puo considerarsi sovmbbondante per i nostri fini.

In efletti, come potra controllarsi a posteriori, nel mentre che il reicolo compie la sua traiettoria, la luna descrive un areo limitato del suo moto di rivoluzione attorno alla terra. Ricordando, difatti, che un mese lunare consta di rentinove gione e assumendo, compatibilmente con le velocita della fase gravitazionale (che salamno di qui a poco calcolate), una duata di tre giomi per il pereorso terra-luna, risulta che durante il tragitto del veicolo, la luna descrive un arco di reirea $25^{\circ}$.

D'altro canto la traiettoria in buona pante non si discostera da ma kepleriana geocentrical. Quindi, i precedenti $25^{\circ}$ verranno ad essere per la maggior parte descritti in condizioni in cui la luna non esercita ma sensibile influenza sullat tracttoria.

In definitiva, mentre la luma influenza la traiettoria, essa descrivera solo un pirecolo arreo.

Queste considerazioni ei sospingono ad adottare la seguente approssimazione: considerare la luna ferma (rispetto alla terra) nella posizione finale che assumerebbe al termine della durata prescritta per il tragitto ("*).

In queste ipotesi, rispetto ad una terma greocentrica (non solidale alla terra) con lasse $x$ diretto verso la posizione in cui si suppone ferma la luna, le equazioni del moto si scrivono (indicate con gli indici 1 e 2 grandezze riferite alla terra e alla luma, rispettivamente):

$$
\begin{aligned}
m \frac{x^{2} x}{d t^{2}}= & -G m_{1} m \frac{x-r_{1}}{\left[\left(x-x_{1}\right)^{2}+\left(y-y_{1}\right)^{2}+\left(z-z_{1}\right)^{2}\right]^{3 / 2}}- \\
& -G m_{2} m \frac{x-x_{2}}{\left.\left[x-x_{2}\right)^{2}+\left(y-y_{2}\right)^{-}+\left(z-z_{2}\right)^{2}\right]^{3 / 2}}
\end{aligned}
$$

(*) Con ejo si trascurera la sghembita finale della traiettoria, la quale, viceversa, va tenuta accuratamente in conto in sede di guida. 


$$
\begin{aligned}
m \frac{d^{2} y}{d t^{2}}= & -\left(r m_{1} m \frac{y-y_{1}}{\left[\left(x-x_{1}\right)-\left(y-y_{1}\right)^{2}+\left(z-z_{1}\right)^{2}\right]^{3 / 2}}-\right. \\
& -\left(i m_{2} m \frac{y-y_{2}}{\left[\left(x-x_{2}\right)-\left(y-y_{2}\right)^{2}+\left(z-z_{2}\right)^{2}\right]^{3 / 2}}\right. \\
m \frac{d-z}{d t^{2}}= & -G m_{1} m \frac{z-z_{1}}{\left[\left(x-x_{1}\right)^{2}+\left(y-y_{1}\right)^{2}+\left(z-z_{1}\right)^{2}\right]^{3 / 2}}- \\
& -G m_{1} m \frac{z-y_{2}}{\left[\left(x-x_{2}\right)^{2}+\left(y-y_{2}\right)^{2}+\left(z-z_{2}\right)^{2}\right]^{3 / 2}}
\end{aligned}
$$

Eliminata $m$ ed introdotta una funzione $V$ defunita come seguc:

$$
\begin{aligned}
V & =\frac{\left(r m_{1}\right.}{\left[\left(x-x_{1}\right)^{2}+\left(y-y_{1}\right)^{2}+\left(z-z_{1}\right)^{2}\right]^{1 / 2}}+ \\
& +\frac{\left(z m_{2}\right.}{\left[\left(x-x_{2}\right)^{2}+\left(y-y_{2}\right)^{2}+\left(z-z_{2}\right)^{2}\right]^{1 / 2}}=G\left[\frac{m_{1}}{r_{1}}+\frac{m_{s}}{r}\right],
\end{aligned}
$$

le equazioni si scrivono in forma piu concisa:

$$
\begin{aligned}
& \ddot{x}=\frac{d V}{d x} \\
& \ddot{y}=\frac{d V}{d y} \\
& \ddot{z}=\frac{d T}{d z} .
\end{aligned}
$$

Sulle [2.5], [2.6] e [2.7] a piu agevole la ricerea di un integrale primo della $[2.1]$, [2.2] e [2.3].

Moltiplicando le [2.5], [2.6] a [3.T] per , $x, y, z$, rispettivamente esommanclo, si ha:

donde:

$$
\left.\frac{d}{d t} \mid \frac{1}{2}\left(r^{2}+y^{2}+z^{2}\right)\right]=\frac{d J^{2}}{d t},
$$

$$
\frac{1}{2} \mathrm{~T}^{2}=\left(i \frac{m_{1}}{r_{1}}+\frac{m_{2}}{r_{2}} \mid-h\right.
$$

Ia [2.9] vale quale che sia la geometria della trabettoria e si presta a considerazioni molto importanti ai fini che qui interessano.

$V^{2}$ non puo diventare negativo. Occore quindi che il secondo mentbro della [2.9] si mantenga durante la traiettoria essenzialnente position.

Cominciamo col prendere in esame l'unica traiettoria di cui si conosca la geometria: quella lettilinea, lungo la congiungente i centri della terra e della luna. 
Tale traiettoria non e certo quella che puo essere descritta dal veicolo, ma, come si dimostrera, gode della proprieta che, le grandezze che interessano, assumono in corrispondenza di essa i loro valori minimi.

Con riferimento alla traiettoria rettilinea detta $d(=384.000 \mathrm{~km})$ la distanza media terra-luna, la [2.9] si scrive:

$$
V=2 G\left[\frac{m_{1}}{x}+\frac{m_{0}}{d-x}\right]-k \quad(k=2 h)
$$

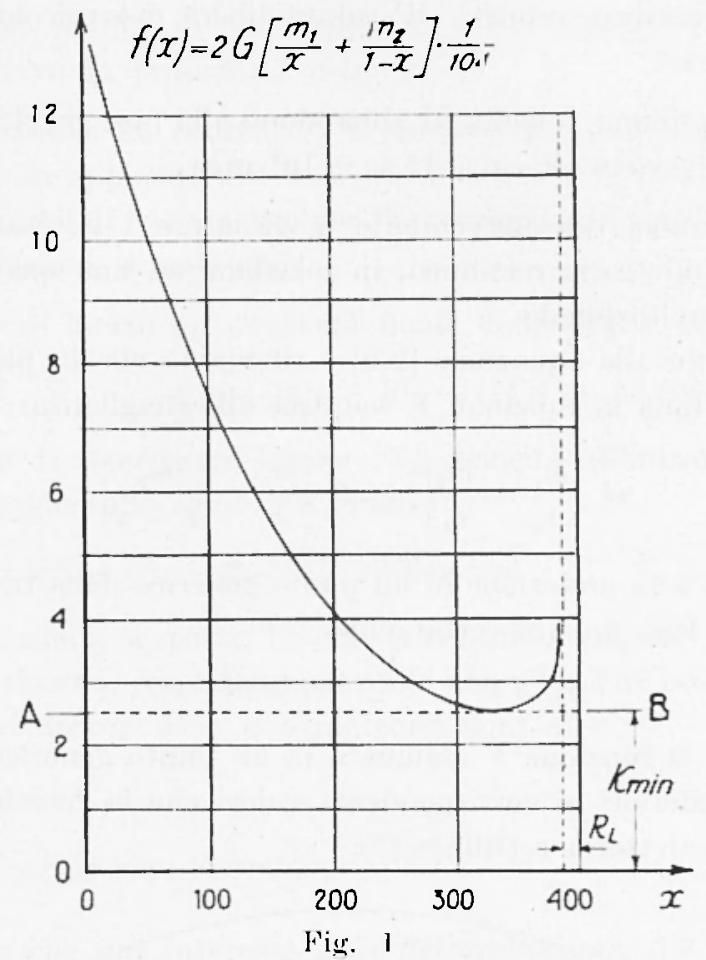

Rappresentiamo nel diagramma di Fig. 1 la funzione:

$$
2\left(\frac{m_{1}}{x}+\frac{m_{2}}{\boldsymbol{d}-x}\right]
$$

ove:

$G$, costante gravitazionale universale $=\frac{6,67}{10^{11}} \frac{\mathrm{ml}^{\mathrm{s}}}{\mathrm{kg}_{\mathrm{m}} \mathrm{sec}^{2}}$

$m_{1}$, massa della terra $=5,975 \times 10^{24} \mathrm{~kg}_{\mathrm{m}}$

$m_{2}$. massa della luna $=7,5 \quad \times 10^{22} \mathrm{~kg}_{\mathrm{m}}$. 
Da questo diagramma si rileva che, se non si vuole che il moto diventi retrogrado nel tratto tra la terra e la luma, il massimo valore che la costante $k$ puo assumere e espresso da:

$$
2,50 \cdot 10^{6} \frac{\mathrm{m}^{2}}{\mathrm{sec}^{2}} \text {. }
$$

Jeggendo le ordinate del diagramma a partire dalla nuova fondamentale AB, si traggono le due seguenti importanti conclusioni:

a) La minima velocita di caduta libera del reicolo sulla luna e circa $2350 \mathrm{~m} / \mathrm{sec}$.

b) Ia minima velocita di abbandono alla fase gravitazionale dopo il lancio deve essere di circa $11,1 \times 10^{3} \mathrm{~m} / \mathrm{sec}$.

Solfermiamoci ora brevemente a discutere i risultati precedenti, relativi alla traiettoria rettilinea, in relazione ad una qualsivoglia altra traicettoria gravitazionale.

Ritorniamo alla equazione [2.9] e rileviamo cle in prossimita della terma e della luna la funzione $V$ soddisfa all'eguaglianza:

$$
G\left[\frac{m_{1}}{r_{1}}+\frac{m_{2}}{r_{2}}\right]=G\left|\frac{m_{1}}{x}-\frac{m_{2}}{d-x}\right|,
$$

mentre, detta $x$ la proiezione di un punto generico della traiettoria schematizzata in Fig. 3, sara certamente:

$$
r_{1} \geqslant x ; \quad r_{2} \geqslant(d-x) \text {. }
$$

Pertanto la funzione $T$ assumera in un punto generico della traiettoria valori inferiori ai corrispondenti valori che la funzione $T$ assume nel caso di traiettoria rettilinea (*).

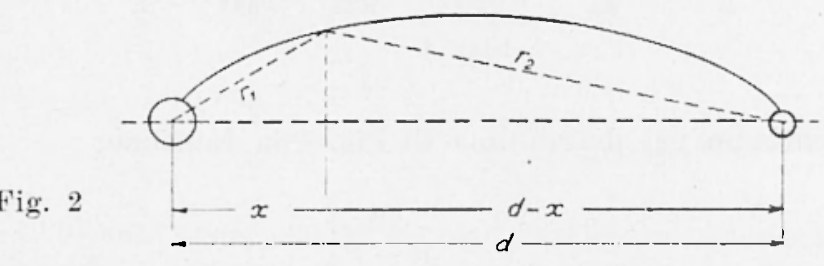

Quindi il diagramma della funzione $F$, analogo a quello di Fig. 2, tracciato per la generica traiettoria (in funzione, at esempio dellascissa

$\left(^{*}\right)$ Al limite, vi potrebbero essere valori uguali nel caso che la traiettoria curva intersecasse in qualche punto quella rettilinea. 
curvilinea) presenterebbe gli stessi valori della traiettoria rettilinea agli estremi e valori inferiori nei punti intermedi. Cio comporterebbe un valore ammissibile minore per li (cioe, sul diagramma, un abbassamento della fondamentale $A B$ ) e quindi valori della velocita agli estremi maggiori di quelli relativi alla traiettoria rettilinea.

Dall'analisi precedente restano dunque chiariti:

a) il legame esistente tra le condizioni iniziali e finali della fase gravitazionale, espresso dalla (2.9);

b) l'impossibilita di abbassare oltre il limite di $2300 \mathrm{~m} / \mathrm{sec}$ la velocita di arrivo in prossimita della luna.

Inoltre l'analisi ha consentito di determinare quali siano le condizioni ai limiti da applicare alle velocita al termine della fase di lancio e all'inizio della fase di frenamento per l'atterraggio sulla superficie lunare.

Tali condizioni si scrivono:

a) fase di lancio ( $V_{L}$ velocita finale della prima fase propulsa):

$$
V_{o}=O \quad V_{L}=\left(x^{2}{ }_{L}+y^{{ }^{2}}+z_{L}\right)^{1 / 2} \cong 11.000 \mathrm{~m} / \mathrm{sec}
$$

b) Fise di atterraggio lunare ( $V_{a}$, velocita all'inizio della manovia di atterraggio sulla superficie lunare):

$$
V_{a}=\left(x^{2}{ }_{a}+y^{2}{ }_{a}+\dot{z}_{a}^{2}\right)^{1 / 2} \geqslant 2300 \mathrm{~m} / \mathrm{sec} \quad V_{f}=0 .
$$

Tali condizioni, a parte l'ovvia inversione, valgono anche per la traiettoria di ritorno, con riferimento alle due altre fasi propulse: decollo dalla luma (condizione b) e rientro (condizioni $a$ ).

\section{3. - STUtolo Delde Fast proputse.}

L'aspetto che qui interessa e la determinazione del "rapporto di massa " tra la massa iniziale e la massa finale de] reicolo, relativamente ad una fisse propulsa.

d) Fase di lancio.

I) Influenza del momento della quantita di moto dovuto alla rotazione della terra.

La traiettoria risultera generalmente curva. Non sono, infatti, nemmeno teoricamente realizzabili traiettorie rettilinee, per la presenza di un 
momento della quantita di moto da cui e affetto il missile all'istante de' suo lancio, per essere, prima di questo, solidale con la terra.

Tale momento della quantita di moto, calcolato rispetto al centro della terra, vale, per unita di massa:

$$
\mathrm{K}_{1}=\text { (1) } R^{2} T
$$

ove:

$$
\text { (1) }=2 \pi \frac{\text { ridd }}{\text { giolmo }} \quad \text { e } \quad h_{T}=6370 \mathrm{~km}
$$

e non sara istantaneamente amullabile se non applicando un momento esterno infinito.

11) Discussione della traiettoria ascensionale.

Se si assume una terma di riferimento solidale alla terra, sono possibili traiettorie ascensionali apparentemente rettilinee (trascurando la variazione della componente della velocita derivante dalla conservazione del momento della quantita di moto, di cui alla [3.1]).

Quindi sono considerabili le usuali tratettorie apparenti di lancio, rostituite da un tratto rettilineo rui si raccorda un tratto curvo.

L'equazione della traiettoria rettilinea ascensionale, seritta in forma che consenta di esaminame la struttuma matematica e la seguente:

$$
z+f(z, z, t) z^{2}+g(z, t)=0
$$

avendo indicato con $z$ l'ascissa lungo la traiettoria.

Tale equazione non consente speranze di soluzione in forma chiusa e va integrata numericamente, con lo svantaggio di dover procedere per tentativi nell'assegnare la portata massima $\dot{m}$ (vedi [3.3]).

L'impossibilita di una risoluzione in forma chiusa deriva essenzialmente dal termine

$$
f(z, z, t) z^{-}
$$

che rappresenta la resistenza aerodinamica.

Ora, però, sofiermiamoci a fare qualche considerazione che, pure nell'oscurita della soluzione generale della [3.2] consenta tuttavia di arclarare qualche elemento.

Poiche, come è a priori intuibile e come calcoli preliminari dimostrano, verremmo a trovarei in un campo inusitato di ordini di grandezan per le variabili in groco, a il caso di effettuare un controllo relativo all'importanza dei varii termini dellequazione di equilibrio. 
Questa, nei simboli usuali, si scrive:

$$
m g(z)+\operatorname{Cros} V^{2}+m V e=m \frac{d V^{r}}{d t} \quad(m<0)
$$

oppure, in termini di accelerazioni:

$$
\frac{d V}{d t}=-g(z)-\frac{\left(V_{0} N V^{2}\right.}{m}+\frac{T}{m} .
$$

Assumendo per $m$ un ordine di grandezza delle migliaia di tomnellate, da controllarsi a posteriori, si ha dumante la fase atmosferica:

- Ordine di grandezza di $g(z)=10 \mathrm{~m} / \mathrm{sec}^{2}$

- Ordine di grandezza di $|T / m|>10 \mathrm{~m} / \mathrm{sec}^{2}$ (per esempio $\left.25 \mathrm{~m} / \mathrm{sec}^{2}\right)$.

- Ordine di grandezza di $\frac{C r Q N V^{2}}{m}=2.5 \mathrm{~m} / \mathrm{sec}^{2}$.

Difatti, assumendo i seguenti ordini di grandezza per i valij fattori della resistenza:

$C r=0,1 ; \quad \varrho=\frac{1}{10} \frac{\mathrm{kg}_{\mathrm{f}} \mathrm{sec}^{2}}{\mathrm{mt}} ; S=\pi r^{2}=\pi \cdot 4^{2}=50 \mathrm{~m}^{2} ; V^{2}=10^{3} \mathrm{~m} / \mathrm{sec}$ si ha:

$$
\frac{\left(r \varrho S V^{2}\right.}{m}=\frac{5 \cdot 10^{6}}{2 \cdot 10^{6}} \cdot \frac{N}{k \cdot g_{m}}=2,0 \mathrm{~m} / \mathrm{sec}^{2}
$$

Quindi, nel campo delle costruzioni in esame, il contributo all'acceleazione del mobile doruto alla resistenza aerodinamica risulta, anche per valutazioni abbastanza accurate, trascurabile, essendo dellordine dei metri per sece rispetto alle diecine di metri per sece cui da luogo la differenza tra gli altri termini.

Taa soluzione della [3.4], negligendo il termine dovuto alla ressistenza e assumendo una spinta costante, risulta dopo semplici calcoli:

$$
r_{1}=r_{e} l n \frac{m_{n}}{m_{0}+m t}-y_{m} t .
$$

Detta to la dumata della fase propulsa, si ha dalla [3.5) l'espressione del rappolto di massa nella fase di lancio:

$$
\mu=\frac{m_{a}}{m_{0}+\dot{m} t_{p}}=\exp \left|\frac{V_{1}}{V_{e}}, \frac{g t_{p}}{V_{e}}\right| .
$$

Alla tractetoria quasi rerticale nel moto relatiro alli tera, corrisponde una traiettoria curva: la rotazione della terla contribuisce a direzionalizzare il moto. 
B) Fasi di atteraggio sulla superficie lumare e di decollo da essata.

Il problema dell'attermagrio non distrut tivo e, implicitamente, quello del decollo dalla superficie lumare es stato gia studiato (). Di questa ricerea si riportano qui brevenente i risultati principali.

Le equazioni del moto rispetto al riferimento intrinseco della traiettoria sono le seguenti (Fig. 3):

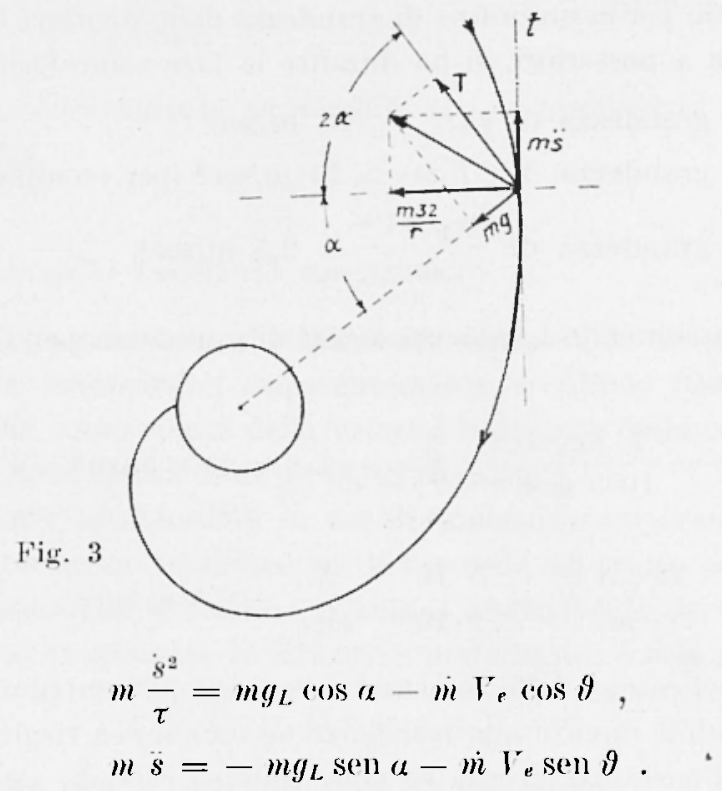

Dalla seconda di queste equazioni si trace:

$$
\frac{i n}{m}=-\frac{s}{V \cdot \sin \vartheta}-\frac{g_{L}}{V_{e}} \frac{\operatorname{sen} \ell}{\operatorname{sen} \vartheta}
$$

donde:

$$
\ln \frac{m_{f}}{m_{i}}=-\frac{1}{r_{e}} \int_{i_{i}}^{t_{f}} \frac{s d t}{\operatorname{sen} \vartheta}-\frac{g_{L}}{r_{e}} \int_{t_{i}}^{t_{f}} \frac{\operatorname{sen} \theta}{\operatorname{sen} \vartheta} d t
$$

Nel lavoro citato fu in dettaglio studiata la fase di attermagio lungo una traiettoria avente la geometria di ma spirale logaritmica. In questo (aso $a==$ cost e si puo porre $\theta=$ cost, e la [3.10] diviene:

$\ln \frac{m_{e}}{m_{i}}=-\frac{i}{V_{e} \sin \vartheta}|s|_{l_{i}}^{t_{f}}-\frac{g_{L} \operatorname{sen} \iota}{T_{e} \sin \vartheta}|t|_{t_{i}}^{t_{f}}=-\frac{1}{\operatorname{sen} \vartheta}\left[\frac{V_{f}-V_{i}}{V_{e}}+\frac{g_{L} \operatorname{sen} \iota}{V_{e}} t_{p} \mid\right.$ avendo ancora indicato con $t_{p}$ la durata della fase propulsa. 
Dalla [3.11] segue che, purche la manovra non sia di lunga durata, il secondo termine dell'ultimo membio della [3.11] e prevalente rispetto al primo $(*)$. In definitiva il valore minimo del rapporto di massa richiesto si può determinare per difetto dalla [3.8] trascurando il termine gravitazionale e ponendovi $\theta=90^{\circ}$ (spinta tangenziale). Si ha allora:

$$
\left.\frac{1}{\mu}=\frac{m_{f}}{m_{i}}=\exp \mid-\frac{V_{i}}{V_{e}}\right\rceil
$$

supposta $r_{e}=3500 \mathrm{~m} / \mathrm{sec}$, si lia:

$$
\frac{1}{\mu}=\frac{m_{f}}{m_{i}}=\exp [-0,5 \tau]=\frac{1}{1.75} .
$$

Considerazioni del tutto analoghe portano ad attribuire jere la fase di decollo lo stesso valore del rapporto di massa.

\section{(1) Fetse di lancio.}

In questa delicatissina fase il veicolo si presenta con un'energia (inetioa per unita di massa, ammontante, secondo la |2.9] a $6,1 \cdot 10^{\text {z }}$ Joule $/ \mathrm{kg}$.

Cerchiamo ora di orientarci circa le modalita della traiettoria di rientro. Appare evidente la necessita di evitare al veicolo e all'equipaggio, gia sottoposti durante la missione a duri cimenti, pericolose sollecitazioni inerziali e termiche.

Cio porta alla considerazione di una frenatura extra atmosferica funo a valori della velocita tali da evitare condizioni di volo ipersoniche nellarcos atmosferico della traiettoria di rientro.

Rimandando ad altri studi per la determinazione delle caratteristiche dinamiche e cinematiche di ma siffatta tratettoria, facciamo adesso le seguenti assunzioni che non dovebbero alterare sostanzialmente $i$ valori delle grandezze che interessa conoscere:

- La traiettoria di arvicinamento del veicolo alla superficie terrestre, rispetto ad una tema luotante solidalnente alla terra, abbia un andamento non molto discosto da quello rettilineo.

- Si realizzi una frenatura extra-atmosferica che piduca la velocita del reicolo sino a circa $800 \mathrm{~m} / \mathrm{sec}$ intorno ai $50 \mathrm{~km}$ di quota.

(*) Difatti, supponendo la durata della fase propulsa pari a tre minuti primi, si laa:

$$
\begin{aligned}
& V_{i} \cong 2000 \mathrm{~m} / \mathrm{sec} \\
& q_{L} \text { sen } \alpha t p<g_{L} t_{p}=1,75 \mathrm{~m} / \mathrm{sec}^{2} \times 180 \mathrm{sec} .=31+\mathrm{m} / \mathrm{sec} .
\end{aligned}
$$


- Si sappia realizzare una traiettoria non distruttiva, planata 0 semplicemente frenata con mezzi aerodinamici, per un missile abbandonato nelle condizioni di cui al punto precedente in prossimita degli strati densi dell'atmosfera.

Supposte soddisfatte queste condizioni le equazioni della frenatuma per la fase di rientro si ridurono, mutatis mutandis, alle [3.5] [3.6].

\section{4. - RApPORTI DI Massi Nelle fasi propulse.}

Designando con $\mu_{i}$ ( $\left.i=1,2,3,4\right)$ i rapporti di massa relativi alle quattro fasi propulse, in applicazione delle ipotesi e dei risultati del precedente $\$ 3$ e delle condizioni ai liniti di $§ \stackrel{2}{2}$, si ottiene una prima valutazione dei $\bar{\mu}_{i}$, assumendo un impulso specifico del propellente pari a $300 \mathrm{~m} / \mathrm{sec}$ :

1a fase:

$$
\bar{\mu}_{1}=\cdots \exp (3,9)=50
$$

2a fase:

$$
\bar{\mu}_{2} \cdots\left({ }^{2}\right)(0,53)=1.7
$$

$3^{\text {a fase: }}$

$$
\bar{\mu}_{3}=\exp (0,53)=1.7
$$

$4^{\text {a fase: }}$

$$
\mu_{4}=(\times x)(3,2)=25
$$

\section{5. - LINEAMENTI DEL, PRogetTo DEL VeICOLO COMPLETO.}

In linea generale, entro (amp)i di variazione non troppo ampi della massa di propellente $m_{c}$ e della spinta, si puo ritenere che la massa della struttura $m_{s}$ sia legata alle due precedenti gramdezze dalla seguente relazione:

$$
m_{s}=r m_{i}+r_{1} \frac{T}{g} .
$$

Sel campo di costruzioni in studio sembra lecito ammettere che il secondo termine del secondo membro della [5.1] sia trascurabile rispet to al primo; inoltre la pratica costruttiva sovietica ed americana ha mostrato rhe:

è uII valore scattabile.

$$
\cdot=0,1
$$


Indichiamo ora con $m_{i, r}$ la massa di un missile all'inizio della combustione dell' $(r+1)$-esimo starlio e cerchiamo di stabilire un'espressione della massa di propellente dell'r-simo stadio in fumzione del rapporto di massa relativo allo stadio $r$-esimo e della massa $m_{i, r \div 1}$.

Si ha:

$$
\begin{aligned}
\mu_{r}=\frac{m_{i, r}}{m_{s . r}} & =\frac{m_{1, r}+1+m_{s, r}+m_{s, r}}{m_{i, r+1}+m_{s, r}}=\frac{m_{c, r}}{m_{i, r}+1+m_{s, r}}+1= \\
& =\frac{m_{c, r}}{m_{i, r-1}+r m_{c}}+1,
\end{aligned}
$$

dontle:

$$
\cdots r_{,}=\frac{\mu_{r}-1}{1-r\left(\mu_{r}-1\right)} m_{t, r+1}
$$

(2:

$$
\frac{m_{s, r}}{m_{r, r+1}}=\frac{r\left(\mu_{r}-1\right)}{1-r\left(\mu_{r}-1\right)}=f\left(\mu_{r}\right)
$$

Effettuiano adesso la determinazione della massa iniziale di un missile a tre stadi, per ciascuno dei quali sia stato previamente determinato il rapporto di massa $\mu_{r}$ (incliei $i$ ed $f$ per condizioni iniziali e finali rispettivamente).

L'algoritmo e il seguente:

$$
\begin{aligned}
& m_{1,4}=m_{u} \\
& m_{,, 3}=m_{u}+m_{s, 3}=m_{u}+f\left(\mu_{3}\right) \cdot m_{u} \\
& m_{i, 3}=\mu_{3} \cdot m_{i, 3}=\mu_{3} m_{u}+\mu_{3} f\left(\mu_{3}\right) \cdot m_{u} . \text { ecc. }
\end{aligned}
$$

Ij: lgoritmo precedente si generalizza nella seguente formula:

$$
\mu=\sum_{l=1}^{J=n} \mu_{i}\left[1+f\left(\mu_{l}\right)\right]
$$

ove col simbolo $\pi$ si indiea il prodotto di 1 fattori e $f\left(\mu_{j}\right)$ e la funzione definita dalla [5.t].

Lat [5.7] a una formula fondamentale per il progetto di un veicolo di assegmato carico utile $m_{u}$.

Un'importante osservazione va fatta sulla espressione [5.3] della massa di propellente necessaria a produrre una. prestazione definita dal valore del rapporto di massial $\mu$ :

$$
m_{c}=\frac{\mu-1}{1-r(\mu-1)} m_{n} .
$$


II dover essere la massa $m_{c}$ non negativa impone che sia:

$$
1-r(\mu-1) \geqslant 0 \text {. }
$$

La precedente relazione definisce un valore limite del rapporto di massa espresso da:

$$
\mu_{\mathrm{inn}}=\frac{1}{r}+1
$$

Per $r=0,1$, secondo l'indicazione relativa allo stato attuale della tecmologia, la [6.3] definisce il seguente valore limite del rapporto di massa:

$$
\mu_{\lim }=\frac{1}{\hat{v, i}}+1==11 \text {. }
$$

D'altra parte il valore $\mu_{\text {im }}$ espresso dalla [6.3] rappresenta di per sé un valore inaccessibile, poiche ad esso corrisponde un valore infinito della massa di propellente.

In definitiva, per ogni stadio r-esimo occorre rhe sia:

$$
\mu_{r}<\mu_{\mathrm{im}}
$$

\section{6. - Critert APplichtTII.}

Tenendo in conto la limitazione enunciata nel precedente paragrafo, riesaminiamo i valori numerici del $\S 4$.

Da quest'esame risulta immediatamente che non e, nemmeno in linea di principio, possibile rhe le due fasi propulse da effettuarsi in prossimita della terra possano realizzarsi mediante l'uso di un solo stadio per' riasceuna di esse.

Quindi per una missione completa che si svolga secondo le modalita illustrate al $\S 1$, occorre riferirsi ad un vettore a numero di stadi non inferiore a sei.

Studiamo ora come vada adeguato il progetto ai dati del $\S 4$. Riprendiamo la relazione [3.6], nella quale in sede di calcoli orientativi negligeremo il termine $g \frac{t_{b}}{\mathrm{~V}_{e}}$ e che quindi, si scrive:

$$
\ddot{\sim j}-\frac{m_{i}}{m_{f}} \quad \cdots\left|\frac{Y_{1}}{V_{\boldsymbol{e}}}\right|
$$

Un esame del significato della [6.6] mostra ches, se $\mu_{j}>\mu_{\text {lim }}$, per realizzare al termine della $j$-esima fase propnlsa la velocita $F_{1}$, occorre $e$ 
basta che, detto s il numero di stadi con cui si realizza detta fase e $\mu_{j, t}$ il rapporto di massa relativo al $t$-esimo di questi stadi, sia:

$$
\bar{\mu}_{j}=\pi_{i=1}^{t \cdots s} \mu_{j: t}
$$

ove $\bar{\mu}_{J, t}$ e il rapporto di massa per la fase jesinal, alcolato al $\$ 3$ ed esemplificato al $\$ 4$.

Ad esempio, supposta realizzata la prima fase mediante l'uso di due stadi deve essere $(\$ t)$ :

$$
\mu_{1}=50=\mu_{1,1} \cdot \mu_{1,2} .
$$

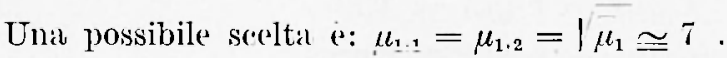

Naturalmente la scelta della coppia di valori soddisfacenti la [6.7] va fatta in relazione a criteri di sicurezza del volo negli strati densi dell'atmosfera e di ottimalizzazione del rapporto di massa globale.

Occorre notare che quando $\mu_{j}>\mu_{\text {lim }}$ e quindi e necessario scindere la fase -esima in piu stadii, il rapporto di massa effettivo relativo all"intera fase $j$-esima risulta maggiore di quello teorico $\bar{\mu}_{j}$. Cio risulta immediatamente dal confronto tra la [5.7] e la [6.7].

\section{7. - DETERMINAZIONI YUMERICHE.}

Riservandoci di applicare in m'ulteriore ricerca dei processi di ottimalizzazione in merito al problema della ripartizione del veicolo in stadi, effettuiamo adesso delle prime determinazioni numeriche, tenendo pero in conto la limitazione, espressa dalla [5.9] delle masse dei singoli stadi.

Riferiamoci ad un missile avente il minimo numero possibile di stadi, cioe a sei stadi. Assumiamo per le fasi iniziale e finale i seguenti valori dei rapporti parziali di massa $(\$+)$ :

$$
\begin{aligned}
& \mu_{1,1}=\mu_{1,2}=\sqrt{\bar{\mu}_{1}} \cong 7 \\
& \mu_{4,1}=\mu_{4,2}=\sqrt{\bar{\mu}_{4}} \bumpeq 5 .
\end{aligned}
$$

Per il vettore considerato assumiamo un rapporto strutturale $r$ eguale per tutti gli stadi e pari a $1 / 10$. 
I rapporti di massa per i singoli stadi, attribuendo man numerazione progressiva sono dunque:

$$
\begin{aligned}
& \mu_{1}=\bar{i} \\
& \mu_{2}=i \\
& \mu_{3}=1.7 \\
& \mu_{1}=1.7 \\
& \mu_{5}=5 \\
& \mu_{i \mathrm{i}}=5
\end{aligned}
$$

Si laa inoltre:

$$
\begin{aligned}
& f\left(\mu_{1}\right)=f\left(\mu_{2}\right)=1,5 \\
& f\left(\mu_{3}\right)=f\left(\mu_{1}\right)=0,075 \\
& f\left(\mu_{5}\right)=f\left(\mu_{6}\right)=1,665 .
\end{aligned}
$$

Appliando la $[5,7]$, tenendo conto delle precedenti nguaglianze, si ot tiene:

$$
\begin{array}{r}
\frac{m_{i}}{m_{n}}=\sum_{j=1}^{j}{ }_{j}^{6} \mu_{j}\left|1+f\left(\mu_{J}\right)\right|=\mu_{1}^{2} \cdot \mu_{2}^{2} \cdot \mu^{2} \mid 1+f\left(\mu_{1}\right)^{2} . \\
\left.\cdot \mid 1+f\left(\mu_{2}\right)\right]^{2} \cdot\left[1+f\left(\mu_{3}\right)\right]^{2}=8+\cdot 10^{3} .
\end{array}
$$

\section{8. - CONSIDERTZIONL SUI RISLITATI.}

I risultati stabiliti mettono in risalto l'importanza tecenica delle considerazioni che seguono.

a) Indaghiamo l'influenza del rapporto struttumale r sul rapporto di

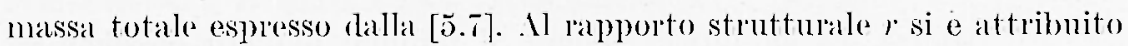
un valore costante e pari ad 1/10. Tale valore deve ritenersi gia noterolmente basso. Tuttavia calcoliamo il limite del repporto totale ri massa per $r-\rightarrow 0$.

Tale valore limite e espresso semplicemente da:

$$
\mu^{*}=\sum_{J=1}^{J=n} \mu_{J}
$$

e, nel caso specifico, particolarizzando la [7.1], si ha:

$$
\mu^{*}=\mu_{1}^{\dot{1}_{1}} \cdot \mu_{2} \cdot \mu^{2}{ }_{3}=3700 \text {. }
$$


In tali condizioni limiti, per ogni kg di carico rientrante, basterebbe predisporre all'istante del lancio 3,7 tomellate in logogo delle 80 calcolate in base alla [7.1].

La riduzione dei rapporti strutturali a valori minori del $10 \% \mathrm{rap}$ presenta quindi l'obbiettivo fondamentale, dal punto di vista delle strutture della tecnologia.

b) Per costruzioni molto spinte quali quelle richieste per una simile missione, in relazione soprattutto all'atterraggio su un suolo aceidentato quale quello della superficie lunare, l'ordinaria disposizione in stadi disposti l'uno dopo l'altro, dovra essere probabilmente abbandonata, ricomendo ad aceoppiamenti del tipo di quello esemplificato in Fig. $t$ a coppie concentriche di stadi.

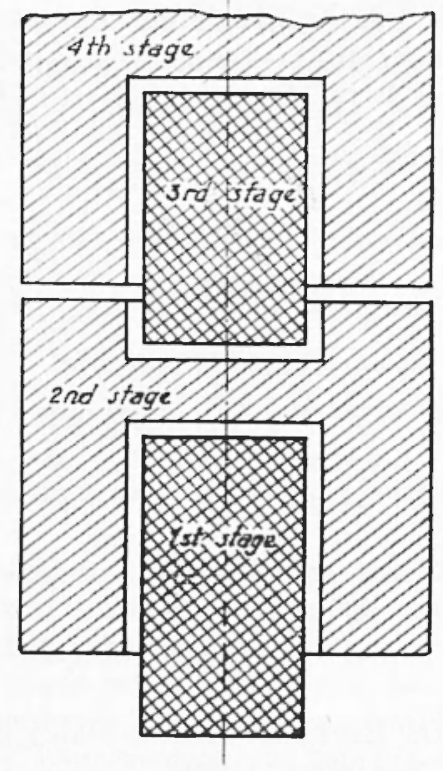

Fig. 4

c) La seoraggiante entita del rapporto globale di massa richiesto per ma simile missione arablora la tecniche di "rendez-vous", verso le quali sembla orientata la tecnica americana e russa, in relazione al tipo di missione lumare fiu impegnativo, cioe quello comportante l'invio di esseri unani. Questa tecnica, estremamente difficile, presenta il fondamentale vantaggio di comportare, nelle varie fasi $n$ reicoli di dimensioni piu lidotte. 


\section{Conchusioxi.}

Nel presente lavoro, acclarata l'importanza dello studio delle fasi gravitazionali in relazione al dimensionamento del vettore, si e svolta una trattazione approssimata delle medesime che ha consentito la determinazione delle condizioni iniziali e finali per le velocita nelle fasi propulse.

Si e quindi studiata la dinamica delle fasi propulse, allo scopo di pervenire, avvalendoci anche dei risultati di un precedente lavoro, ad espressioni del rapporto di massa in funzione delle condizioni ai limiti dianzi determinate.

Si sono poi discussi i criteri di ripartizione del missile in stadi, sottolineando talune limitazioni di principio nelle prestazioni dei razzi nronostadi e stabilendo delle utili formule di progetto.

In applicazione dei precedenti risultati si sono effettuate delle prime determinazioni numeriche. Queste metiono in risalto l'importanza di alcune considerazioni svolte al $\S 7$.

\section{BIBI_IOGRAFIA}

(1) Zuenek Kopat. The moon. Chapman and Mall, London, 1960.

${ }^{(2)}$ U.S.S.R. Acadeny of Sciences, The other face of the moon. Pergamon Press, 1960.

${ }^{(3)}$ United States Information Service, Satelliti artificiali statunitensi. Roma, 1952 .

(') G. Contursi, Sui sistemi di allemaggio non distrultivi di un missile abitato sulla superficie lunare. "Missili ", 1, (1962).

(5) L'URSS' e lo Spazio. Scritti e documenti ufficiali sovietici. Lerici editore, Milano, 1960.

${ }^{(6)}$ Wrobec J., Breshearsi R., Lunar landing vehicle propulsion requirements A.R.S. "Journal ", November, (1961).

(7) Fincay-Freunducir E., Gelestial Mechanies. Pergamon Press, London, (1958). 